\title{
Reynolds stresses and meridional circulation from rotating cylinder simulations
}

\author{
C. Hupfer ${ }^{1}$, P. J. Käpylä ${ }^{1,2,3}$, and M. Stix ${ }^{1}$ \\ 1 Kiepenheuer-Institut für Sonnenphysik, Schöneckstr. 6, 79104 Freiburg, Germany \\ e-mail: hupfer@kis.uni-freiburg.de \\ 2 Observatory, University of Helsinki, PO Box 14, 00014 University of Helsinki, Finland \\ 3 Astronomy Division, Department of Physical Sciences, PO Box 3000, 90014 University of Oulu, Finland
}

Received 28 April 2006 / Accepted 4 August 2006

\section{ABSTRACT}

\begin{abstract}
Aims. The latitude and depth dependences of Reynolds stresses are obtained from numerical simulations of a solar-type convection zone where the star is assumed to rotate with a uniform angular velocity.

Methods. A two-dimensional model, using a cylindrical annulus with axis perpendicular to the axis of rotation, is introduced as an approximation of a spherical section along the meridional plane. We solve the fully compressible Navier-Stokes equations numerically and use Cartesian and cylindrical geometries to simulate convection under the influence of rotation.

Results. For moderate Coriolis numbers both models yield strong extrema of the Reynolds stress component $Q_{\theta \varphi}$ at low latitudes near the equator, and a meridional cell pattern is found in the cylindrical model. For Coriolis numbers larger than about 10 the flow becomes aligned with the direction of the rotation axis.
\end{abstract}

Key words. hydrodynamics - convection - Sun: rotation

\section{Introduction}

The differential rotation of the Sun and solar-type stars is still under examination both in observation and theory. There are three contributions capable of redistributing the angular momentum in a convection zone: meridional circulation, turbulent transport, and magnetic fields. In the present work the treatment is restricted to the non-magnetic case, mainly based on the assumption that the magnetic field in the present Sun has no great effect on the internal rotation - this may not be true for young stars, and therefore for the young Sun as well, e.g. Pinsonneault et al. (1989).

In spherical coordinates $(r, \theta, \varphi)$ the equation of angular momentum transport reads, see e.g. Rüdiger (1989),

$$
\frac{\partial}{\partial t}\left(\rho \Omega s^{2}\right)+\boldsymbol{\nabla} \cdot\left(\rho \Omega s^{2} \boldsymbol{u}_{\mathrm{m}}-\rho s^{2} v \boldsymbol{\nabla}_{\mathrm{m}} \Omega\right)=0
$$

where $\Omega, v, s=r \sin \theta$, and $\boldsymbol{u}_{\mathrm{m}}=\left(u_{r}, u_{\theta}, 0\right)^{T}$ denote the angular velocity, kinematic viscosity, lever arm, and meridional velocity, respectively, and axisymmetry is assumed; $\boldsymbol{\nabla}_{\mathrm{m}}=\boldsymbol{e}_{r} \frac{\partial}{\partial r}+\boldsymbol{e}_{\theta} \frac{1}{r} \frac{\partial}{\partial \theta}$ is a meridional gradient.

Since turbulent diffusion is much greater than molecular diffusion, a statistical approach via mean-field theory is appropriate, where the time evolution of, say, the mean velocity $\langle\boldsymbol{u}\rangle$ is calculated using the averaged Navier-Stokes equation, which is often called the Reynolds equation. Averaging of the equation of angular momentum transport introduces the Reynolds stress tensor $Q$ as a possible generator of differential rotation (Rüdiger 1989). The Reynolds stresses are defined as

$Q_{i j}=\left\langle u_{i}^{\prime} u_{j}^{\prime}\right\rangle$,

where the brackets denote averaging and $u_{i}{ }^{\prime}=u_{i}-\left\langle u_{i}\right\rangle$ is the fluctuation of the $i$ th velocity component. For the mean angular momentum transport equation one obtains (neglecting the stress due to molecular diffusion)

$$
\frac{\partial}{\partial t}\left(\rho \Omega s^{2}\right)+\boldsymbol{\nabla} \cdot\left(\rho \Omega s^{2}\left\langle\boldsymbol{u}_{\mathrm{m}}\right\rangle+\rho s\left\langle u_{\varphi}^{\prime} \boldsymbol{u}_{\mathrm{m}}^{\prime}\right\rangle\right)=0,
$$

which contains the Reynolds tensor components $Q_{r \varphi}$, responsible for radial, and $Q_{\theta \varphi}$, responsible for horizontal transport of angular momentum. If this transport is from higher to lower latitudes towards the equator, then $Q_{\theta \varphi}>0$ on the northern hemisphere of the Sun. Similarly, $Q_{r \varphi}>0$ means outward transport of angular momentum.

The quantity $Q_{\theta \varphi}$ has been measured, for example, by Ward (1965) from the motion of sunspot groups, by Schröter \& Wöhl (1976) from the $\mathrm{Ca}^{+}$network, and more recently by Vršnak et al. (2003) also for higher latitudes from coronal bright points. Typical values for $Q_{\theta \varphi}$ are of the order $1000 \mathrm{~m}^{2} \mathrm{~s}^{-2}$. The experimental determination of the horizontal correlation is difficult due to the large rotation velocity $u_{\varphi} \approx 2000 \mathrm{~m} \mathrm{~s}^{-1}$, compared to the meridional velocity $\left|u_{\theta}\right| \approx 10 \mathrm{~m} \mathrm{~s}^{-1}$. There is no observational information on $Q_{r \varphi}$, nor on the depth dependence of both correlations. Reynolds stress values for other stars are unknown.

Meridional circulation appears in both forms of the angular momentum transport equation. It seems to be confirmed by helioseismology that there is a meridional circulation pattern down to a depth of at least $15000 \mathrm{~km}$ (Zhao \& Kosovichev 2004). Direct observation of the surface flows yields results indicating a northward flow on the northern hemisphere of the order of $-10 \mathrm{~m} \mathrm{~s}^{-1}$. From recent observations of K-type giants (Weber et al. 2005) there are indications of strong meridional surface velocities, of order $1000 \mathrm{~m} \mathrm{~s}^{-1}$, and anti-solar differential rotation. 


\subsection{The closure model of Boussinesq and the $\Lambda$ effect}

From a theoretical point of view it is difficult to calculate Reynolds stresses due to the fact that the derived expressions generally involve higher order correlations. This gives rise to the closure problem which requires that the higher order terms need to be related to lower order correlations or mean quantities in order to make the equations tractable. An early ansatz comes from Boussinesq (1897):

$Q_{i j}=-v_{\mathrm{t}}\left(\frac{\partial\left\langle u_{i}\right\rangle}{\partial x_{j}}+\frac{\partial\left\langle u_{j}\right\rangle}{\partial x_{i}}\right)$

Using this in spherical coordinates one obtains

$Q_{r \varphi}=-v_{\mathrm{t}} r \sin \theta \frac{\partial \Omega}{\partial r}$,
$Q_{\theta \varphi}=-v_{\mathrm{t}} \sin \theta \frac{\partial \Omega}{\partial \theta}$.

These expressions resemble the terms in Eq. (1) if the molecular diffusion $v$ is replaced by the turbulent diffusion $v_{\mathrm{t}}$; but the Boussinesq terms neither provide generation of differential rotation if the star is rotating uniformly initially, nor can they maintain the angular velocity gradient against the strong diffusion implied by the turbulent viscosity in a star which already rotates differentially. Moreover, the observed signs of $Q_{\theta \varphi}$ and $\partial \Omega / \partial \theta$ are incompatible with Eq. (6). There must be an additional term that is non-diffusive and acting even in a uniformly rotating star. The non-diffusive term is usually called the $\Lambda$-effect. It can be written (Rüdiger 1989) as

$Q_{r \varphi}=-v_{\mathrm{V}} r \sin \theta \frac{\partial \Omega}{\partial r}+\Lambda_{\mathrm{V}} \Omega \sin \theta$,

$Q_{\theta \varphi}=-v_{\mathrm{H}} \sin \theta \frac{\partial \Omega}{\partial \theta}+\Lambda_{\mathrm{H}} \Omega \cos \theta$,

where a distinction between a vertical (radial) and horizontal $\Lambda$ effect with correspondingly different turbulent viscosities $v_{\mathrm{V}}, \nu_{\mathrm{H}}$ has been made.

\subsection{Numerical approaches}

Reynolds stresses have been determined already by Hathaway \& Somerville (1983) with box simulations in the moderate rotation regime at a near-equator latitude; they calculated both $Q_{r \varphi}$ and $Q_{\theta \varphi}$. More recently similar box calculations were performed by Pulkkinen et al. (1993), Chan (2001), Käpylä et al. (2004), Hupfer et al. (2005), and Rüdiger et al. (2005) to explore the Reynolds stresses and the $\Lambda$ effect, and the possibility of a latitude dependency of the turbulent heat transport ("pole-equator temperature difference"). Robinson \& Chan (2001) made simulations in a section of a spherical shell, and global spherical simulations were run by Miesch et al. (2000) and Elliott et al. (2000) with a spherical harmonics code.

In the subsequent two sections we present our models and the equations that have been solved; in Sect. 4 we briefly describe the numerical method. Sections 5 and 6 contain the results and a discussion.

\section{The models}

In this section we briefly describe our two models. Both have an initially piecewise polytropic stratification in hydrostatic equilibrium, which yields constant thermal conduction coefficients

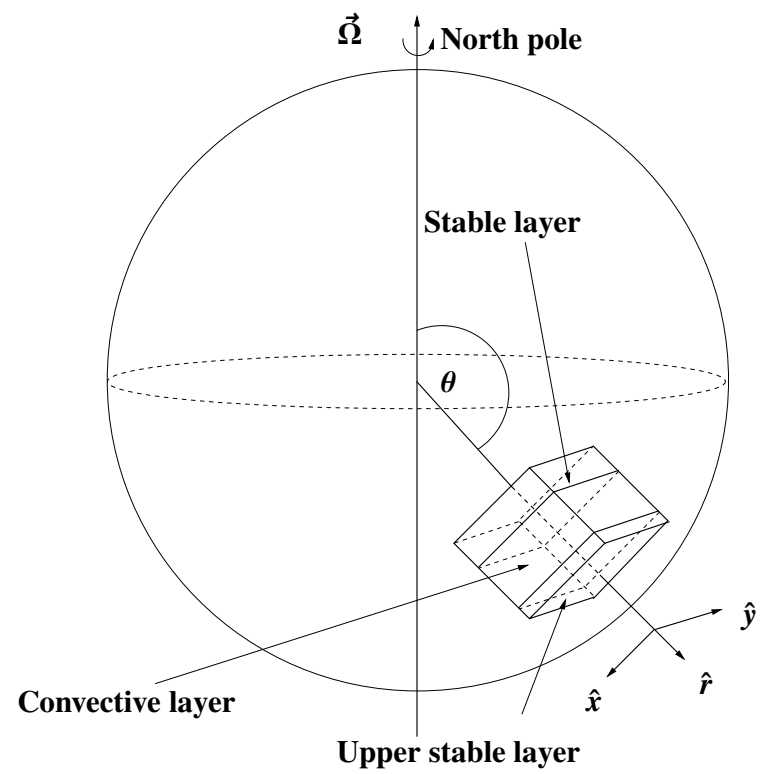

Fig. 1. The rectangular domain of computation, with three layers, here located at an intermediate latitude on the southern hemisphere.

and linear temperature profiles in each layer. The total vertical extent of the box models and the cylinder models CA is 2.65 density scale heights initially; for the cylinder models $\mathrm{CB}$ and $\mathrm{CC}$ it is 2.9 density scale heights.

\subsection{The box model}

The calculations of this model are carried out within a rectangular box that consists of three layers, as shown in Fig. 1. The middle layer, of thickness $d$, is convectively unstable; the lower layer, of thickness $0.68 d$, is stably stratified, but the flow is allowed to overshoot into it from above. The upper layer, of thickness $0.32 d$, is also stable; in Hupfer et al. (2005) we presented results for Reynolds stresses from models in which we cooled this layer effectively, so that it remains nearly isothermal; we considered this upper layer mainly as a technical means to remove the heat that enters the box from below. The present calculations, however, have shown that, at least for the parameter regime under consideration, such cooling is not necessary; it was therefore omitted in the present simulations. In order to explore the influence of rotation we vary the strength of the Coriolis force and place the box at different latitudes. The box is assumed to be small so that the co-moving unit vectors $\hat{\boldsymbol{e}}_{x}, \hat{\boldsymbol{e}}_{y}, \hat{\boldsymbol{e}}_{r}$ are identified with the local spherical coordinate system $\hat{\boldsymbol{e}}_{\theta}, \hat{\boldsymbol{e}}_{\varphi}, \hat{\boldsymbol{e}}_{r}$, respectively. In contrast to some of the above-mentioned publications the vertical axis $\hat{\boldsymbol{e}}_{r}$ has an outward orientation. The layer boundaries are located at $r_{0}=r_{\mathrm{b}}, r_{1}=r_{0}+0.68 d, r_{2}=r_{1}+d, r_{3}=r_{\mathrm{t}}$, where $r_{\mathrm{b}}, r_{\mathrm{t}}$ denote the radial positions of the bottom and the top of the box.

\section{Boundary conditions for the box model}

The horizontal extent of the box is $4 d$. The boundary conditions in the horizontal directions $(x, y)$ are periodic for all variables. The energy flux and thereby the temperature gradient is prescribed at the bottom of the box. Contrary to the simulations presented in Käpylä et al. (2004) or Hupfer et al. (2005) we apply this condition at the top of the box as well, with the same heat flux that enters the box at the bottom leaving it at the top. 


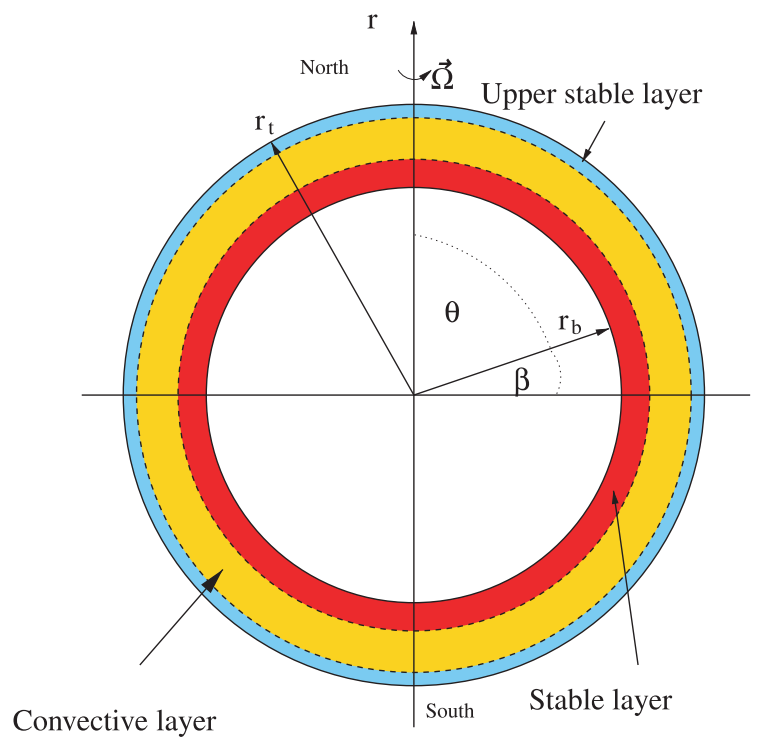

Fig. 2. Rotating cylinder model with three layers. The latitude $\beta=90^{\circ}-$ $\theta$ is measured from the equator, and $r_{b}, r_{t}$ are the radii of the bottom and the top of the vertical computation domain.

The heat conduction coefficients in the lower and upper stable layer are the same, resulting in the same temperature gradient at the vertical boundaries. The box is assumed to be impenetrable in the vertical direction, and for the horizontal velocity we use stress-free boundary conditions:

$\left(u_{r}\right)_{\mid r=r_{\mathrm{b}}, r_{\mathrm{t}}}=0$.

$\left(\frac{\partial u_{x}}{\partial r}\right)_{\mid r=r_{\mathrm{b}}, r_{\mathrm{t}}}=\left(\frac{\partial u_{y}}{\partial r}\right)_{\mid r=r_{\mathrm{b}}, r_{\mathrm{t}}}=0$.

The box model has two notable deficiencies:

- The flows at different latitude are disconnected and each box acts independently.

- The latitude of a box is assumed to be constant. For a solar application, however, $d$ is of order $10^{5} \mathrm{~km}$ and, with a horizontal box size $4 d$, the latitude range covered by a typical calculation is of order $30^{\circ}$.

\subsection{The cylinder model}

In order to compare whether geometry and connected flows influence the results obtained by box calculations we have developed an approach that may be considered as an intermediate step between local and global models: a cylindrical annulus is assumed to represent a spherical section in the $r, \theta$-plane; the cylinder axis is perpendicular to the axis of rotation (Fig. 2). We restrict ourselves to a two-dimensional model, depending on the coordinates $r$ and $\beta$, with 3 velocity components $u_{r}, u_{\beta}, u_{\phi}$. In this setup the azimuthal coordinate of the cylindrical geometry corresponds to the meridional direction $\theta$, and the direction along the cylinder axis serves as an approximation of the spherical $\varphi$ coordinate, however, with sign changes due to the different orientations of the coordinate systems. We use the latitude $\beta$ rather than the co-latitude and the symbol $\phi$ as the axial direction of the cylinder model ( $\phi$ has the dimension of a length). The orientation is such that $\hat{\boldsymbol{e}}_{\theta}=-\hat{\boldsymbol{e}}_{\beta}$ and $\hat{\boldsymbol{e}}_{\varphi}=-\hat{\boldsymbol{e}}_{\phi}$ (eastern hemisphere of the corresponding embedded sphere). Thus, there are sign changes in the velocities as well, namely $u_{\varphi}=u_{y}=-u_{\phi}$ for the azimuthal direction, and $u_{\theta}=u_{x}=-u_{\beta}$ for the meridional direction. The separation into three layers, the intermediate layer boundaries, and the stratification are the same as in the box models. Efficient calculations could be restricted to one quadrant or one hemisphere. However, our simulations cover the full $360^{\circ}$ range, with 721 grid points in the meridional direction and 64 grid points for the radial direction. The advantage of such "global" calculations is the possibility to check for symmetry or anti-symmetry of flows on different quadrants or hemispheres.

Since the thickness of the convective layer is our length scale, of order $10^{5} \mathrm{~km}$ in the deep convection zone, we choose in our cylinder model series CA the radii $r_{\mathrm{b}}=5$ and $r_{\mathrm{t}}=7$, which would correspond to $500000 \mathrm{~km}$ and $700000 \mathrm{~km}$ on the Sun. The radius ratio is therefore $\eta=\frac{r_{\mathrm{b}}}{r_{\mathrm{t}}}=\frac{5}{7}$. The series CA consists of 26 calculations from no rotation to very strong rotation, with Coriolis numbers - defined by (25) below - ranging from 0 to $\approx 40$. In addition, we performed simulations with either different radius ratios $\eta$ (models CAS), or with a convective layer of vertical extent $2 d$ (models $\mathrm{CB}, \mathrm{CC}$ ) in order to compare with the CA results. In these models the upper and lower layers remain unchanged, so that the total depth is $3 d$, cf. Fig. 7 below. An overview of the cylinder models is given in Table 2 .

A deficiency of the cylinder model is that there is no true polar region due to the Cartesian geometry along the cylinder axis. In an axisymmetric sphere there would be a converging or diverging meridional flow at the poles; this is not necessarily the case in the model of a cylinder rotating around an axis perpendicular to its own axis.

\section{Boundary conditions for the cylinder model}

Since the full circle is calculated, the mathematical periodicity assumed for the meridional coordinate $x$ in the box models turns into a physical periodicity - there are no obstacles for a meridional flow, so we are using periodic boundary conditions also in this case. The vertical boundary conditions are the same as for the box model.

\section{Equations and dimensionless parameters}

We use $\frac{D}{D t}=\frac{\partial}{\partial t}+\boldsymbol{u} \cdot \boldsymbol{\nabla}$ for the substantial derivative, and the Einstein summation in the case of index notation. The symbols $\Delta, \boldsymbol{\nabla} \cdot \boldsymbol{A}$, and $\nabla_{k} \Psi$ denote the Laplacian operator, the divergence of a vector $\boldsymbol{A}$, and the $k$ th component of the gradient of a scalar function $\Psi$. We give the hydrodynamical equations for Cartesian and cylindrical geometry separately and use the usual symbols $\rho, e, \boldsymbol{u}$ for the mass density, the energy density and the velocity, respectively. Since the density varies by more than two scale heights over the radial extent of the computational domain it is preferable to use $\ln \rho$ rather than the density itself; this also simplifies the equations and enhances the speed of the computation.

\subsection{Cartesian geometry}

For the box model we use Cartesian coordinates:

$\frac{\mathrm{D} \ln \rho}{\mathrm{D} t}=-\boldsymbol{\nabla} \cdot \boldsymbol{u}$

$\frac{\mathrm{D} e}{\mathrm{D} t}=-\frac{P}{\rho} \boldsymbol{\nabla} \cdot \boldsymbol{u}+\frac{1}{\rho} \boldsymbol{\nabla} \cdot(\kappa \boldsymbol{\nabla} e)+2 v \underline{S}^{2}$,

$\frac{\mathrm{D} u_{i}}{\mathrm{D} t}=-\frac{1}{\rho} \frac{\partial P}{\partial x_{i}}+g_{i}-2(\boldsymbol{\Omega} \times \boldsymbol{u})_{i}+2 v\left(\frac{\partial S_{i j}}{\partial x_{j}}+S_{i k} \frac{\partial \ln \rho}{\partial x_{k}}\right)$, 
where $\underline{S}$ denotes the symmetric strain tensor

$S_{i j}=\frac{1}{2}\left(\frac{\partial u_{i}}{\partial x_{j}}+\frac{\partial u_{j}}{\partial x_{i}}\right)-\frac{1}{3} \delta_{i j} \boldsymbol{\nabla} \cdot \boldsymbol{u}$,

with $S^{2}=S_{i k} S_{i k} ; v$ is the kinematic viscosity, and $\kappa$ the heat conduction coefficient (divided by $c_{\mathrm{V}}$ ). The influence of rotation is given by the Coriolis force $\boldsymbol{F}_{\text {Cor }}=-2 \rho \boldsymbol{\Omega} \times \boldsymbol{u}$, which introduces a dependence on the latitude $\beta$, sometimes called " $\beta$ - effect" in Geophysics:

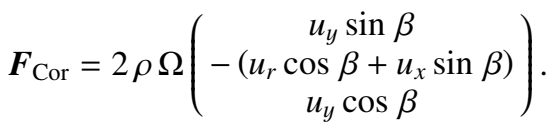

The angle $\beta$ is measured from the equatorial plane to the North pole with positive values, i.e. from $0^{\circ}$ to $90^{\circ}$. The gravity is assumed to have only a radial component, i.e. $\boldsymbol{g}=\left(0,0, g_{r}\right)$.

The conservation equations are supplemented by the equation of state for a perfect gas,

$P=(\gamma-1) \rho e$,

where $\gamma=c_{\mathrm{P}} / c_{\mathrm{V}}=5 / 3$.

\subsection{Cylindrical geometry}

For cylindrical geometry both the mass and energy conservation Eqs. (11) and (12) remain unchanged if one uses vector notation. The three components of the Navier-Stokes equation read:

$$
\begin{aligned}
\frac{\mathrm{D} u_{r}}{\mathrm{D} t}= & \frac{u_{\beta}^{2}}{r}-\frac{1}{\rho} \frac{\partial P}{\partial r}+g_{r}+\frac{1}{\rho}\left(\boldsymbol{F}_{\mathrm{Cor}}\right)_{r}+2 v\left(S_{r k} \nabla_{k} \ln \rho\right) \\
& +v\left[\Delta u_{r}-\frac{u_{r}}{r^{2}}-\frac{2}{r^{2}} \frac{\partial u_{\beta}}{\partial \beta}+\frac{1}{3} \frac{\partial}{\partial r}(\boldsymbol{\nabla} \cdot \boldsymbol{u})\right], \\
\frac{\mathrm{D} u_{\beta}}{\mathrm{D} t}= & -\frac{u_{\beta} u_{r}}{r}-\frac{1}{\rho r} \frac{\partial P}{\partial \beta}+\frac{1}{\rho}\left(\boldsymbol{F}_{\mathrm{Cor}}\right)_{\beta}+2 v\left(S_{\beta k} \nabla_{k} \ln \rho\right) \\
& +v\left[\Delta u_{\beta}-\frac{u_{\beta}}{r^{2}}+\frac{2}{r^{2}} \frac{\partial u_{r}}{\partial \beta}+\frac{1}{3 r} \frac{\partial}{\partial \beta}(\boldsymbol{\nabla} \cdot \boldsymbol{u})\right] \\
\frac{\mathrm{D} u_{\phi}}{\mathrm{D} t}= & -\frac{1}{\rho} \frac{\partial P}{\partial \phi}+\frac{1}{\rho}\left(\boldsymbol{F}_{\mathrm{Cor}}\right)_{\phi}+2 v\left(S_{\phi k} \nabla_{k} \ln \rho\right) \\
& +v\left[\Delta u_{\phi}+\frac{1}{3} \frac{\partial}{\partial \phi}(\boldsymbol{\nabla} \cdot \boldsymbol{u})\right],
\end{aligned}
$$

where

$$
\underline{S}=\left(\begin{array}{lll}
S_{r r} & S_{r \beta} & S_{r \phi} \\
S_{\beta r} & S_{\beta \beta} & S_{\beta \phi} \\
S_{\phi r} & S_{\phi \beta} & S_{\phi \phi}
\end{array}\right)
$$

(note that $\phi$ has the dimension of length). The precise form of the cylindrical stress tensor for fully compressible flow can be found in Appendix A. The Coriolis force in this system reads

$$
\boldsymbol{F}_{\mathrm{Cor}}=2 \rho \Omega\left(\begin{array}{c}
-u_{\phi} \cos \beta \\
+u_{\phi} \sin \beta \\
u_{r} \cos \beta-u_{\beta} \sin \beta
\end{array}\right),
$$

in the order of the unit vectors $\hat{\boldsymbol{e}}_{r}, \hat{\boldsymbol{e}}_{\beta}, \hat{\boldsymbol{e}}_{\phi}$.

\subsection{Parameters}

\subsubsection{Scaling}

Since only hydrodynamics is considered, basic mechanical units are sufficient for the scaling of the equations. We choose $d$ as unit of length, and $g=|\boldsymbol{g}|=\left|g_{r}\right|$ as unit of gravity; it follows that $t_{0}=\sqrt{d / g}$ and $u_{0}=\sqrt{d g}$ are the units of time and velocity. In order to compare our results with solar values we use $g=370 \mathrm{~m} \mathrm{~s}^{-2}$ as a typical value for the gravitational acceleration in the middle of the solar convection zone, and $d \approx 100000 \mathrm{~km}$. This yields $t_{0} \approx 10 \mathrm{~min}$ and $u_{0} \approx 200 \mathrm{~km} \mathrm{~s}^{-1}$, which is of the order of the sound speed at the bottom of the convection zone. The unit of the viscosity is given by $v_{0}=u_{0} d$, the unit of density is $\rho_{1}=\rho\left(r_{1}\right)$, the density at the interface between the lower and middle layers.

\subsubsection{Dimensionless parameters}

A simulation is controlled by the following dimensionless parameters: The Rayleigh number

$\mathrm{Ra}=\frac{L^{4} g \delta}{\chi v H_{\mathrm{P}}}$

the Prandtl number

$\operatorname{Pr}=\frac{v}{\chi}$

and the Taylor number

$\mathrm{Ta}=\left(\frac{2 \Omega L^{2}}{v}\right)^{2}$.

The vertical extent $L$ of the unstable layer, which is used in the definitions of $\mathrm{Ra}$ and $\mathrm{Ta}$, is equal to the length scale $d$ in all models except $\mathrm{CB}$ and $\mathrm{CC}$. The parameter $\delta=1 /\left(m_{2}+1\right)-(\gamma-1) / \gamma=0.1$ is the superadiabaticity of the middle (unstable) layer in the initial state, $m_{2}$ is the polytropic index in the unstable layer, $H_{\mathrm{P}}$ is the pressure scale height in the middle of that same layer, and $\chi=\kappa / \rho_{1}$ is the thermal diffusivity. For our choice of parameters, we have $H_{\mathrm{P}}=0.45 d$ initially, and this remains approximately so during the course of the calculation. The heat conductivity $\kappa$ is also from the middle layer. As far as the rotational influence is concerned, the inverse Rossby or Coriolis number,

$\mathrm{Co}=\frac{2 \Omega d}{u_{\mathrm{rms}}}$,

is of more interest than the Taylor number. In our model Co is a derived quantity. For the box models the chosen parameters yield Co $\approx 4$, which is representative for the lower part of the solar convection zone; for the cylinder models we explored a wider range, from $\mathrm{Co}=0$ to $\mathrm{Co} \approx 40$. From $\mathrm{Ra}, \mathrm{Pr}, \mathrm{Ta}, d, g, \gamma$ and the polytropic indices $m_{1}, m_{2}, m_{3}$ of the three-layer models (see Sects. 2.1 and 2.2) the equation parameters $v, \kappa$ and $\Omega$ are calculated. We fixed $d \equiv 1, g_{r} \equiv-1, \gamma=5 / 3, m_{1}=3, m_{2}=1, m_{3}=3$ for all models. The condition of hydrostatic equilibrium yields the stratification of the initial state. For details of the initial stratification we refer to Brandenburg et al. (1996), Ossendrijver et al. (2001), or Käpylä et al. (2004), where the same three-layer models and parameter systems are used. 
Table 1. Box models. Common parameters for all series are $\mathrm{Ra}=$ $250000, \operatorname{Pr}=0.4$, and $v=5.963 \times 10^{-4} v_{0}$. Due to the CFL condition and the larger grid the time step for BD is smaller than for BC, otherwise these models are identical. The Coriolis number Co is determined according to (25) after the data analysis has been done.

\begin{tabular}{ccccccc}
\hline \hline Model & $\boldsymbol{m}$ & Grid & \multicolumn{1}{c}{ Ta } & Cooling & $\Delta t$ & Co \\
\hline BA & $3,1,0$ & $64^{3}$ & 10000 & yes & 0.004 & $\approx 1$ \\
BB & $3,1,0$ & $64^{3}$ & 325000 & yes & 0.004 & $\approx 4$ \\
BC & $3,1,3$ & $51^{3}$ & 325000 & no & 0.008 & $\approx 4$ \\
BD & $3,1,3$ & $64^{3}$ & 325000 & no & 0.006 & $\approx 4$ \\
\hline
\end{tabular}

\section{Numerics}

The hydrodynamical equations were solved with a semi-discrete approach, using 6th-order-accurate finite-difference approximations for spatial derivatives, and either the Adams-BashforthMoulton 3rd-order predictor-corrector scheme described in Caunt \& Korpi (2001), or the 2N-Runge-Kutta 3rd-order method mentioned in Brandenburg (2003) for the time evolution; the latter method allows larger time steps. In all simulations the time step was determined as the smaller of the diffusive and sound travel time over one spatial step, multiplied by a safety factor 0.2 ("Courant parameter"). For the box models we used the finitedifference code already described in Hupfer et al. (2005); for the cylinder models we used cylindrical coordinates and implemented the corresponding geometry into our code. The module containing the hydrodynamic source terms was completely rewritten for this new geometry.

The simulations where run on the 16-processor Linux Cluster KABUL of the Kiepenheuer-Institut and on private personal computers. For the box models the code was validated by determining critical Rayleigh numbers for the onset of convection, and comparison with results of Gough et al. (1976); moreover, comparison simulations were made by M. Ossendrijver using a code derived from one originally written by A. Brandenburg, and by W. Dobler using the Pencil code. No such tests were possible for the cylinder models: we know of no other such models (with cylinder axis perpendicular to the axis of rotation). A measure of the statistical error in box-model results is the behavior of $Q_{\theta \varphi}$ at the pole $\left(\beta=90^{\circ}\right)$; we verified that integrating for a longer time decreased this error. But again this is not true for the cylinder models where $Q_{\theta \varphi}$ generally has a finite value at the pole.

\section{Results}

For both the box and the cylinder models we performed simulations, varying the input parameters. Table 1 summarizes the box models. Each series consists of calculations on different latitudes either on the southern (BA, BB) or on the northern hemisphere. We were interested especially in the behavior of the Reynolds stresses near the equator, where we did additional simulations in small steps of $\Delta \beta=2.5^{\circ}$.

The parameters of the cylinder models are summarized in Table 2. Table 3 lists the variation of the Taylor number for the series CA. The values of $u_{\mathrm{rms}}$ as well as the values of $Q_{\theta \varphi}$ listed in that table clearly demonstrate the increasing rotational effect at moderate Coriolis number, and the quenching at large Coriolis number. The models of the series CAS are identical to the CA models, except for different radii $r_{\mathrm{b}}$ and $r_{\mathrm{t}}$, as listed in Table 4 . Tables 5 and 6 contain models where the thickness of the convectively unstable layer is $2 d$, for two values of the Rayleigh number.
Table 2. Cylinder models. The Prandtl number is always $\operatorname{Pr}=1$, the polytropic indices are identical to those used in the box models BC and $\mathrm{BD}$. The quantity $L$ is the thickness of the convective layer.

\begin{tabular}{cccccc}
\hline \hline Model & Grid & Ra & $L$ & $v\left[v_{0}\right]$ & $\Delta t$ \\
\hline CA & $64 \times 721$ & $10^{6}$ & $d$ & $4.714 \times 10^{-4}$ & 0.005 \\
CB & $96 \times 721$ & $16 \cdot 10^{6}$ & $2 d$ & $3.333 \times 10^{-4}$ & 0.004 \\
CC & $96 \times 721$ & $8 \cdot 10^{6}$ & $2 d$ & $4.714 \times 10^{-4}$ & 0.004 \\
\hline
\end{tabular}

Table 3. Cylinder model series CA, with $\mathrm{Ra}=10^{6}, \mathrm{Pr}=1.0$. The value for $Q_{\theta \varphi}$ is calculated from volume and time averaging over the unstable region, the latitude range $0-35^{\circ}$, and 1500 time units.

\begin{tabular}{rrrrrr}
\hline \hline Model & \multicolumn{1}{c}{$\mathrm{Ta}$} & $\Omega\left[\Omega_{0}\right]$ & \multicolumn{1}{c}{ Co } & $u_{\mathrm{rms}}\left[u_{0}\right]$ & \multicolumn{1}{c}{$Q_{\theta \varphi}\left[u_{0}^{2}\right]$} \\
\hline CA01 & 0 & 0.000 & 0.00 & 0.069 & 0.00000 \\
CA02 & 100 & 0.002 & 0.07 & 0.069 & 0.00003 \\
CA03 & 500 & 0.005 & 0.15 & 0.066 & 0.00001 \\
CA04 & 1000 & 0.007 & 0.22 & 0.066 & -0.00001 \\
CA05 & 2000 & 0.010 & 0.29 & 0.072 & 0.00003 \\
CA06 & 10000 & 0.023 & 0.64 & 0.075 & -0.00011 \\
CA07 & 20000 & 0.033 & 0.83 & 0.081 & -0.00029 \\
CA08 & 25000 & 0.037 & 0.94 & 0.081 & -0.00001 \\
CA09 & 30000 & 0.041 & 0.96 & 0.084 & -0.00006 \\
CA10 & 50000 & 0.052 & 1.17 & 0.090 & -0.00006 \\
CA11 & 100000 & 0.075 & 1.39 & 0.111 & -0.00020 \\
CA12 & 250000 & 0.118 & 1.81 & 0.135 & 0.00039 \\
CA13 & 325000 & 0.134 & 2.17 & 0.126 & 0.00049 \\
CA14 & 400000 & 0.149 & 2.56 & 0.120 & 0.00058 \\
CA15 & 450000 & 0.158 & 2.83 & 0.114 & 0.00063 \\
CA16 & 500000 & 0.167 & 3.03 & 0.111 & 0.00079 \\
CA17 & 550000 & 0.175 & 3.50 & 0.102 & 0.00070 \\
CA18 & 600000 & 0.182 & 4.06 & 0.090 & 0.00037 \\
CA19 & 750000 & 0.204 & 5.23 & 0.078 & 0.00036 \\
CA20 & 800000 & 0.211 & 5.86 & 0.072 & 0.00023 \\
CA21 & 1000000 & 0.236 & 7.49 & 0.063 & 0.00023 \\
CA22 & 1500000 & 0.289 & 10.70 & 0.054 & 0.00020 \\
CA23 & 2000000 & 0.333 & 13.89 & 0.048 & 0.00007 \\
CA24 & 4000000 & 0.471 & 24.15 & 0.039 & -0.00006 \\
CA25 & 6000000 & 0.577 & 32.06 & 0.036 & -0.00005 \\
CA26 & 8000000 & 0.667 & 40.40 & 0.033 & -0.00004 \\
\hline
\end{tabular}

\subsection{Averaging method}

As a number of previous studies have shown, the Reynolds stresses are extremely fluctuating quantities in space and time. The momentary change in time does not reveal anything but turbulence. Since differential rotation is a long term phenomenon, averaging both in space (horizontal coordinates) and time is done. We used up to 1500 individual snapshots to calculate time averages, equally spaced by one time unit for the cylinder models, and by up to four time units for the box models. Since the cylinder simulations use only two coordinates, no horizontal average is performed in order to keep the information on the latitudinal dependence - the results presented in the corresponding figures are time averages only. Sliding averages over a latitude range do not yield significantly different results and are not presented here.

\subsection{Results for $Q_{\theta \varphi}$}

In Table 7 we compare the box models BB, BC, BD with results published in other papers. The common result is that there is a peak of $Q_{\theta \varphi}(r)$ near the surface of the convective layer and at low latitude, near the equator. The latitude of this peak seems to be $\beta_{\max }=10^{\circ} \pm 2.5^{\circ}$. In Fig. 3 we show the depth dependence 
Table 4. Models CAS - cylinder runs with different radius ratios. The models CAS03, CAS04, CAS05 are identical to CA12, CA14, CA16 and are included here for completeness. The angular velocities can be found in Table 3 for the respective Taylor numbers.

\begin{tabular}{rrrcccc}
\hline \hline Model & $r_{b}[d]$ & $r_{t}[d]$ & $\mathrm{Ta}$ & $\mathrm{Co}$ & $u_{\mathrm{rms}}\left[u_{0}\right]$ & $Q_{\theta \varphi}\left[u_{0}^{2}\right]$ \\
\hline CAS01 & 2.0 & 4.0 & 250000 & 1.57 & 0.1500 & 0.00025 \\
CAS02 & 2.0 & 4.0 & 500000 & 2.91 & 0.1140 & 0.00061 \\
CAS03 & 5.0 & 7.0 & 250000 & 1.81 & 0.1350 & 0.00039 \\
CAS04 & 5.0 & 7.0 & 400000 & 2.56 & 0.1200 & 0.00058 \\
CAS05 & 5.0 & 7.0 & 500000 & 3.03 & 0.1110 & 0.00079 \\
CAS06 & 7.0 & 9.0 & 250000 & 2.07 & 0.1180 & 0.00036 \\
CAS07 & 7.0 & 9.0 & 400000 & 3.10 & 0.0960 & 0.00055 \\
CAS08 & 7.0 & 9.0 & 500000 & 4.18 & 0.0780 & 0.00033 \\
\hline
\end{tabular}

Table 5. Models CB - cylinder runs with a convective layer of thickness $2 d$ and Rayleigh number $16 \times 10^{6}$.

\begin{tabular}{ccrrrr}
\hline \hline Model & $\mathrm{Ta}$ & $\Omega\left[\Omega_{0}\right]$ & $\mathrm{Co}$ & $u_{\mathrm{rms}}\left[u_{0}\right]$ & \multicolumn{1}{c}{$Q_{\theta \varphi}\left[u_{0}^{2}\right]$} \\
\hline CB01 & 4000000 & 0.083 & 2.12 & 0.169 & -0.00016 \\
CB02 & 6000000 & 0.102 & 2.47 & 0.165 & 0.00030 \\
CB03 & 7000000 & 0.110 & 2.70 & 0.165 & 0.00063 \\
CB04 & 8000000 & 0.118 & 3.00 & 0.158 & 0.00052 \\
\hline
\end{tabular}

Table 6. Models $\mathrm{CC}$ - cylinder runs with a convective layer of thickness $2 d$ and Rayleigh number $8 \times 10^{6}$.

\begin{tabular}{rcrrrr}
\hline \hline Model & Ta & $\Omega\left[\Omega_{0}\right]$ & Co & $u_{\mathrm{rms}}\left[u_{0}\right]$ & $Q_{\theta \varphi}\left[u_{0}^{2}\right]$ \\
\hline CC01 & 4000000 & 0.118 & 2.73 & 0.173 & 0.00054 \\
CC02 & 4500000 & 0.125 & 2.96 & 0.169 & 0.00051 \\
CC03 & 5000000 & 0.132 & 3.29 & 0.160 & 0.00077 \\
\hline
\end{tabular}

Table 7. Position of the maximum of $Q_{\theta \varphi}$ from box calculations, and comparison with other authors. All results are adapted to the northern hemisphere. The depth position is indicated by $\mathrm{CL}=$ cooling layer, $\mathrm{UCL}=$ upper convective layer, $\mathrm{BCL}=$ bottom of convective layer.

\begin{tabular}{crc}
\hline \hline Model & $\left.\beta_{\max }{ }^{\circ}\right]$ & Depth position \\
\hline BB & 7.5 & CL \\
BC & 10.0 & UCL \\
BD & 7.5 & UCL \\
\hline (Pulkkinen et al. 1993) & $\approx 20$ & BCL \\
(Chan 2001) & $\approx 11$ & UCL \\
(Käpylä et al. 2004, Co = 10) & 7.5 & CL \\
\hline
\end{tabular}

of $Q_{\theta \varphi}$ for boxes placed at different latitudes from model BD. The latitudes from $2.5^{\circ}$ to $20^{\circ}$ dominate obviously over higher latitudes and the equator itself. The latitude and depth dependence from model BD is - apart from the different hemisphere similar to that presented in Fig. 3 in Hupfer et al. (2005), which was produced with data from model BB. The main differences between BB and BD are slightly smaller amplitudes and the fact that all peaks are shifted into the convective layer for the latter model. These differences must be due to a slightly different stratification and a modified heat transport in the upper stable layer, which was not cooled in model BD.

In order to compare the results from box and cylinder simulations it is important to use models which have about the same Coriolis number. We have done box simulations up to $\mathrm{Co} \approx 4$. These are compared with results from the moderate rotation regime, $\mathrm{Co} \approx 1.8$ to $\mathrm{Co} \approx 4.0$, of our cylinder models (models CA12 to CA18 from Table 3). A typical behavior of $Q_{\theta \varphi}(r)$ for different latitudes on the northern hemisphere (first quadrant)

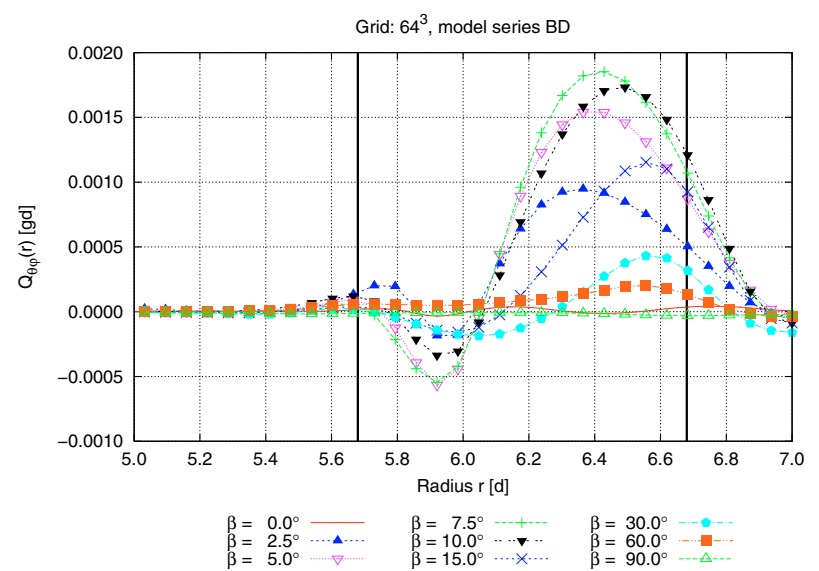

Fig. 3. Reynolds stress component $Q_{\theta \varphi}$ for different northern latitudes as a function of depth from the box model $\mathrm{BD}$, with $\mathrm{Co} \approx 4$. The vertical lines give the boundaries of the unstable layer. (This and the following figures are available in color in electronic form.)

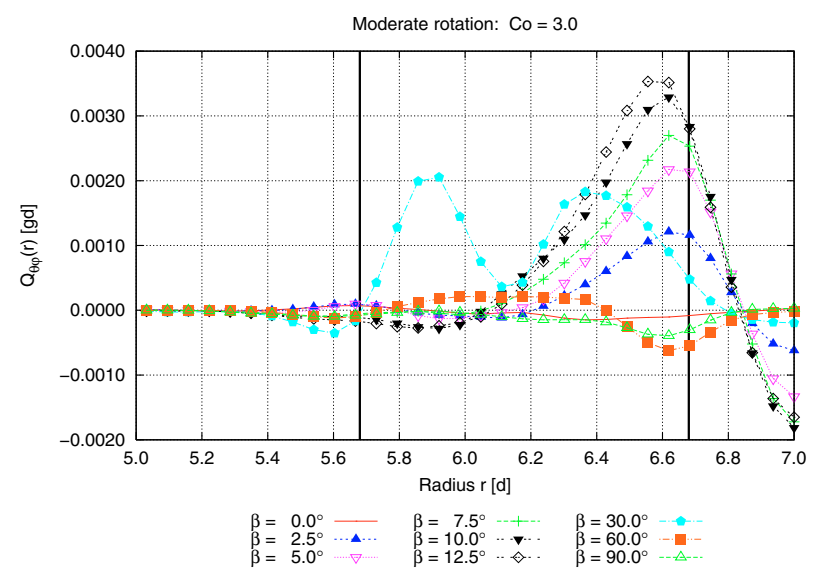

Fig. 4. As Fig. 3, but for the cylinder model CA16, with $\mathrm{Co} \approx 3$. The largest peak appears at $\beta=12.5^{\circ}$.

is shown in Fig. 4 for model CA16, with a maximum peak at $\beta=12.5^{\circ}$.

The amplitude of $Q_{\theta \varphi}(r)$ obtained from cylinder models is always greater than for results from box models. The most probable reason is that the cylinder model has a two-dimensional geometry - there is no third dimension to calculate horizontal averages. Averages over a range of latitudes reduce the amplitudes only slightly. Comparing with Fig. 11 in Käpylä et al. (2004), obtained from box calculations with $\mathrm{Co} \approx 10$, we found no such peak behavior for low latitudes in our cylinder model CA22, which has a similar Coriolis number. In the range $\mathrm{Co}=4$ to 10 the dominant low-latitude peak shifts both to higher latitude, around $\beta \approx 40^{\circ}$, and more into the middle of the unstable layer. When Co exceeds 20 a sign reversal occurs at all latitudes, and the peaks are now minima, located at the top of the convective layer and at $\beta=45^{\circ}$ (Fig. 5). However, the absolute values are smaller by a factor of about five than in model CA16, which indicates quenching of the $\Lambda$ effect, as described by Kichatinov \& Rüdiger (1993). A third dimension used in horizontal averages could also reduce the root mean square velocity $u_{\mathrm{rms}}$, shifting the Coriolis numbers from the cylinder models closer to the values reported by Käpylä et al. (2004).

Apart from smaller amplitudes there is no significant change for $Q_{\theta \varphi}$ if the ratio of the radii is altered. A result for $\mathrm{Co}=2.9$ and $\eta=\frac{2}{4}$ is shown in Fig. 6. There is also no large deviation if 


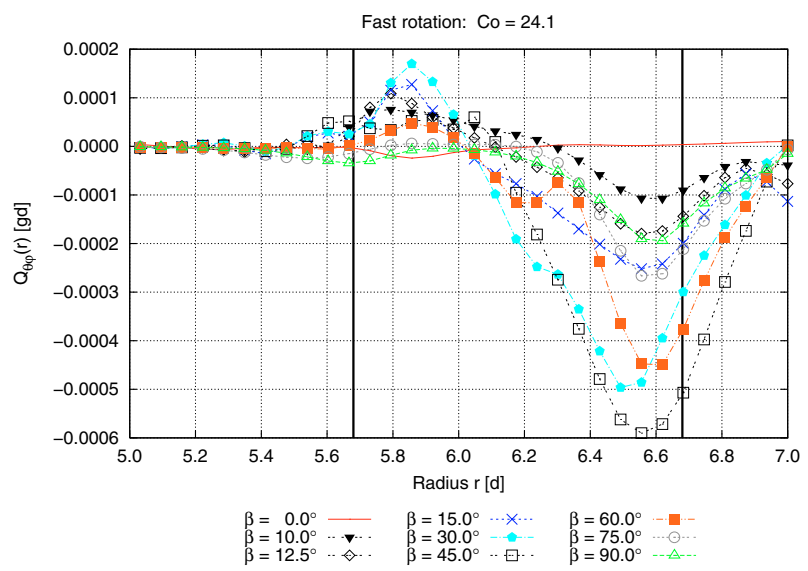

Fig. 5. As Fig. 3, but for the cylinder model CA24 for fast rotation with $\mathrm{Co}=24.1$. The minimum peak is at $\beta=45^{\circ}$. Note the different scaling as compared to Fig. 4.

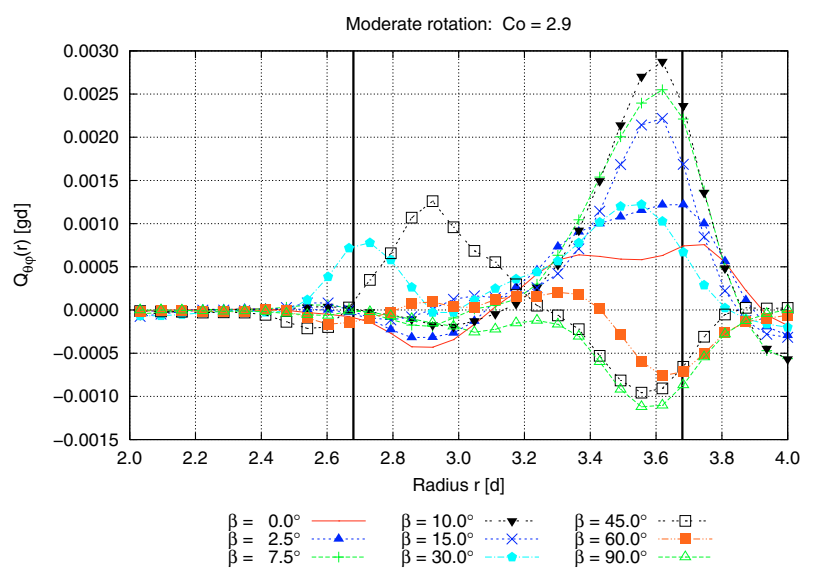

Fig. 6. As Fig. 3, but for the cylinder model CAS02, with $\mathrm{Co}=2.9$. Note the different radii of the lower and upper boundary, as compared to Fig. 4. The largest peak appears at $\beta=10^{\circ}$.

the thickness of the unstable layer is doubled as has been done in the series $\mathrm{CB}$ and $\mathrm{CC}$; the main change consists of broader peaks near the transition region between the convective and the upper stable layer, see Fig. 7. Since both the Rayleigh and the Taylor numbers are proportional to $d^{4}$ it is necessary to adapt both numbers in order to get the same mechanical and thermal viscosities as in the CA cases, otherwise the results would be hard to compare to each other. A common result for all cylinder models is a non-vanishing Reynolds stress component $Q_{\theta \varphi}$ at the poles, $\beta= \pm 90^{\circ}$. This is because in the cylinder models neither $u_{\phi}$ nor $u_{\beta}$ necessarily vanishes at the poles.

\section{Comparison with solar observations}

Ward (1965) determined the correlation $Q_{\theta \varphi}$ from the motion of sunspot groups on the northern hemisphere, with a maximum at $\beta \approx 25^{\circ}$ and $Q_{\theta \varphi} \approx 2000 \mathrm{~m}^{2} \mathrm{~s}^{-2}$. Gilman \& Howard (1984) found similar values from the motion of individual sunspots, with a maximum at $\beta=30^{\circ}$. In a more recent paper Vršnak et al. (2003) published results derived from the motion of coronal bright points, whereby the latitudinal range was extended up to $\beta \approx 60^{\circ}$. They confirmed that $Q_{\theta \varphi}>0$ on the northern hemisphere and the order of magnitude of this correlation, however with large standard deviations (see Fig. $6 a$ and Table 3 in

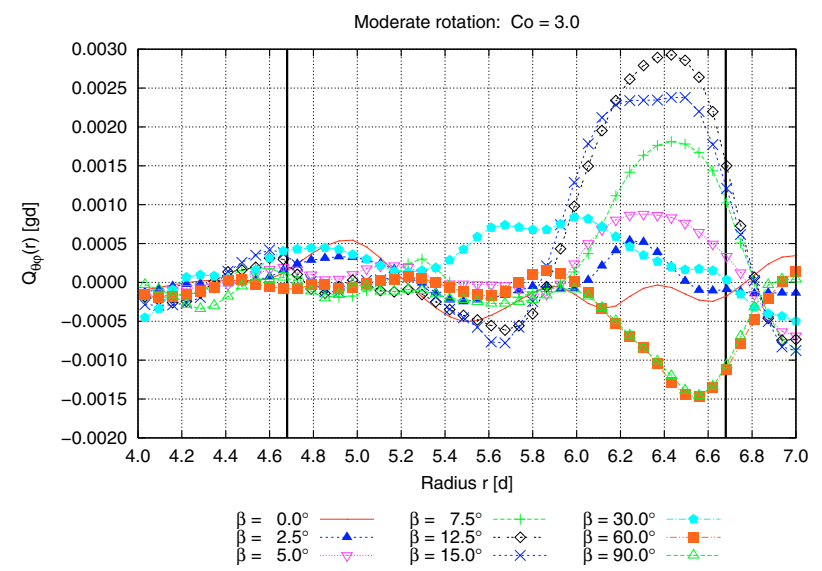

Fig. 7. As Fig. 3, but for the cylinder model CCO2, with $\mathrm{Co}=2.96$. The thickness of the convective layer has been doubled compared to the CA series. See Fig. 4 for differences of this model with CA16 which has virtually the same Coriolis number. The largest peak appears at $\beta=12.5^{\circ}$.

Vršnak et al. (2003); note that this component of the Reynolds stress changes sign when latitude instead of co-latitude is used).

Since $u_{0} \approx 200 \mathrm{~km} \mathrm{~s}^{-1}$ the Reynolds stress components determined by the present numerical models are too large by at least three orders of magnitude if the observed solar surface values are used for comparison. A similar discrepancy occurs if we evaluate the turbulent viscosity according to $v_{\mathrm{t}}=u_{\mathrm{rms}} d / 3$, where $u_{\text {rms }}$ is obtained from a volume and time average over the unstable layer; values are listed in Tables 3 to 6 . These discrepancies are due to the unrealistic parameter regime which is enforced by the constraints of solving the Navier-Stokes equations numerically (essentially the too large input energy flux). Apart from the Coriolis number all other dimensionless parameters given in Sect. 3.3.2 are far away from solar values; e.g., Ossendrijver (2003) lists $\mathrm{Ra}=10^{16} \ldots 10^{20}, \mathrm{Pr}=10^{-7}$, and $\mathrm{Ta}=10^{14} \ldots 10^{27}$ for the Sun. It is therefore only possible to compare the results qualitatively. Both observation and numerical experiment with various codes and geometric setups show that the Reynolds stress $Q_{\theta \varphi}$ is on the whole positive on the northern hemisphere and has a significant peak in the latitude band from $10^{\circ}$ to $30^{\circ}$, with analogous results for the southern hemisphere. However, the numerical results suggest the peak at the transition region between the unstable and the upper stable layer; this is not to be identified with the surface of the Sun if the calculations are considered ro represent the lower half of the convection zone, which is the interesting case because at that depth the rotational influence is significant and the overshooting below the unstable layer is included. Unfortunately, there is no observational information about the dependence of $Q_{\theta \varphi}$ on the depth in the solar convection zone.

The indications for the angular momentum transport are obvious: a positive correlation (on the northern hemisphere) means an equatorward transport, from higher to lower latitudes. The occurrence of the strong peaks in the equator zone should lead also to an enhanced transport of angular momentum and to an increase of the angular velocity, leaving latitude bands in which the rotation is faster than average, and as well bands in which it is smaller than average. Indeed, Vršnak et al. (2003) presented results for such bands, especially the stronger rotation region between $10^{\circ}$ and $25^{\circ}$, cf. Fig. 1b in Vršnak et al. (2003), which remarkably well coincides with the results from numerical 


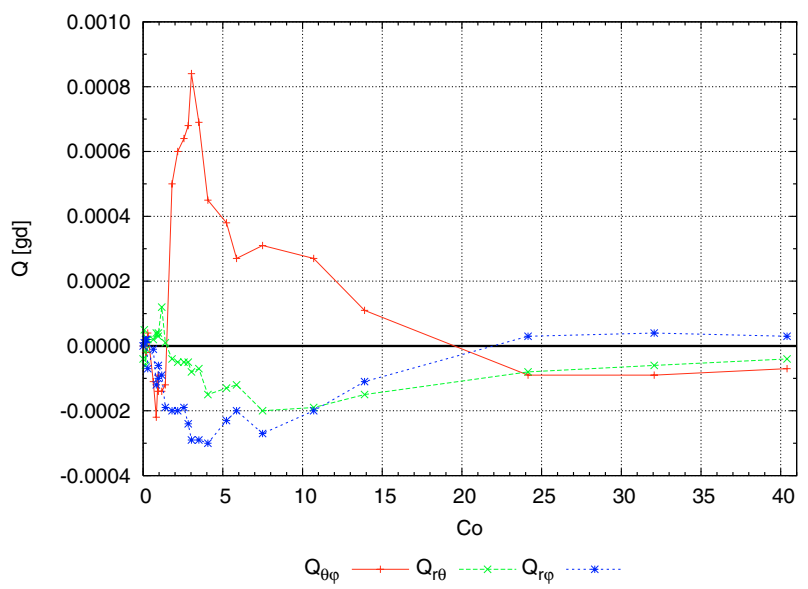

Fig. 8. Reynolds stress components $Q_{\theta \varphi}, Q_{r \theta}, Q_{r \varphi}$, averaged over the range $0-35^{\circ}$ of latitude, for the cylinder models $\mathrm{CA}$ as functions of the Coriolis number.

simulations in this paper or in Pulkkinen et al. (1993), Chan (2001), and Käpylä et al. (2004).

\subsection{Averages over latitude}

In order to show more clearly the qualitative behavior of the Reynolds stresses derived from the cylinder model, we display in Fig. 8 averages over the latitude range $0-35^{\circ}$. This range is probably the most relevant to the Sun; and the cylindrical geometry does not deviate too much from the spherical geometry.

Except for very slow rotation, the horizontal transport is positive in the range of Coriolis numbers relevant to the Sun $(\mathrm{Co} \leq 20)$. As far as the sign is concerned, this is consistent with the result of Kichatinov \& Rüdiger (1993) who used a given anisotropic turbulence model; the magnitude of our result is, however, much larger, for the reasons given above (Sect. 5.2).

The radial transport is negative, directed to greater depths, in about the same range of Coriolis numbers. This is consistent with other recent numerical simulations, e.g. Rüdiger et al. (2005), but in contrast to the earlier analytical result of Kichatinov \& Rüdiger (1993). The meridional correlation $Q_{r \theta}$ is as well negative, as indicated also by the orientation of the circulation pattern.

At very large Coriolis number all three correlations decrease in magnitude, a result that is consistent with Kichatinov \& Rüdiger (1993): very fast rotation quenches the $\Lambda$ effect.

\subsection{Meridional flow}

Information on meridional flow and a possible circulation can be obtained from a global model like the cylinder approach. We found meridional circulation already for the case of no rotation, in the model CA00. In this model the circulation pattern is partitioned into multiple small cells along the meridional direction, having an extent of about $15^{\circ}$. For small Coriolis numbers there is only one cell in the radial direction, concentrated in the convective layer and - due to strong overshooting - in the lower stable layer. As long as Co $<0.6$ the cell near the equator has always a counter-clockwise orientation, i.e. a poleward oriented flow in the upper part of the model. In the range Co $=0.6-1.2$ there is clockwise circulation near the equator, combined with merging of cells, i.e. the number of cells is reduced. For $\mathrm{Co}>1.2$ the cells near the poles vanish and two cells in radial direction

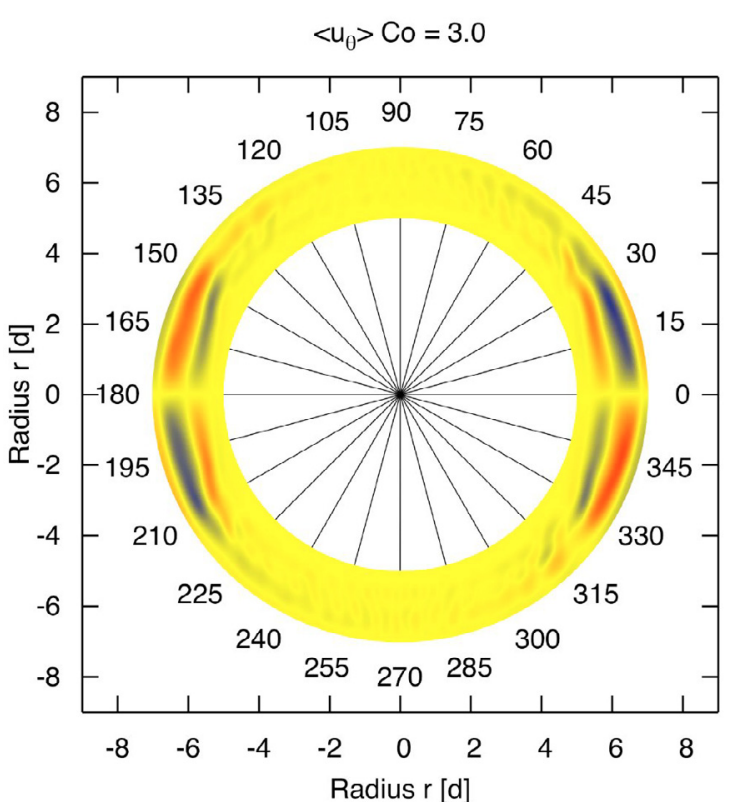

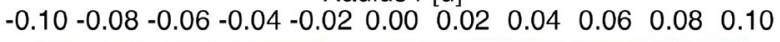

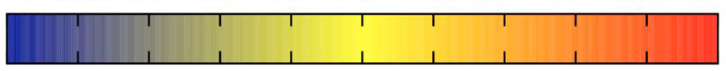

Fig. 9. Contour plot of the mean meridional velocity $\left\langle u_{\theta}\right\rangle$ from the cylinder model CA16. The labels indicate the angles in degrees, counted from the eastern equator. The flow of the dominant cell is directed polewards in the upper, and equatorwards in the lower part of the model.

appear; the outer cell is confined to the upper stable layer and is very thin, its orientation is clockwise, and the flow of the inner cell is contained almost completely within the convective layer, the circulation being counter-clockwise, see Fig. 9. The cells have a latitudinal extent up to $45^{\circ}$; this angle decreases with increasing Coriolis number to about $30^{\circ}$. In the strong-rotation regime $(\mathrm{Co}>4)$ the meridional cell pattern vanishes and we find Taylor columns (Fig. 10) as a consequence of the TaylorProudman theorem, cf. Chandrasekhar (1961).

The box models cover only a narrow range of latitude; nevertheless we may use these models to extract some information on meridional flow, simply by calculation of $\left\langle u_{x}\right\rangle$ for each box. In Figs. 11 and 12 the meridional velocity is shown for the northern hemisphere as obtained from the box models BD and from CA16, a cylinder model with comparable Coriolis number. Positive values indicate an equatorward flow. In Fig. 12 the poleward orientation of the flow in the bulk of the convective layer is clearly discernible, as well as the equatorward orientation at the bottom of the unstable layer and at the top of the computational domain. For the box model a partitioning in (at least) two cells of circulation occurs: the low-latitude cell has a clockwise orientation, whereas the cell at higher latitudes has a counter-clockwise orientation and a smaller magnitude - the lower cell dominates. This is one of the main discrepancies between plane-layer and cylinder calculations. The difference in the form the circulation is probably due to the periodic boundary conditions applied in the meridional direction of the box models.

The circulation pattern vanishes completely if the Coriolis number exceeds a value of $\approx 4$ which also seems to be the limit for the existence of the peak in the Reynolds stress $Q_{\theta \varphi}$. This could be a particular feature of the cylinder model; unfortunately we do not know whether in box calculations the circulation also vanishes for Coriolis numbers beyond 10. From box models with the same stratification specifications as have been applied in this paper, Käpylä et al. (2004) in their Fig. 10 for $\mathrm{Co} \approx 10$ show the 
Table 8. Maximum position for $Q_{\theta \varphi}$, as function of Coriolis number Co for moderate rotation (models CA and CAS). The subscripts 1 and 2 indicate maxima near the surface and near the bottom of the convective layer, respectively.

\begin{tabular}{rrrrrrrr}
\hline \hline Model & Co & $\left.\beta_{\max _{1}}{ }^{\circ}\right]$ & $r_{\max _{1}}[d]$ & $Q_{\theta \varphi_{\max _{1}}}[g d]$ & $\left.\beta_{\max _{2}}{ }^{\circ}\right]$ & $r_{\max _{2}}[d]$ & $Q_{\theta \varphi_{\max _{2}}}[g d]$ \\
\hline CA11 & 1.39 & 30.0 & 6.68 & 0.0007 & 45.0 & 5.64 & 0.0008 \\
CA12 & 1.81 & 30.0 & 6.64 & 0.0040 & 46.0 & 5.92 & 0.0020 \\
CA13 & 2.17 & 30.0 & 6.60 & 0.0028 & 41.5 & 5.92 & 0.0020 \\
CA14 & 2.56 & 12.5 & 6.60 & 0.0028 & 42.5 & 6.08 & 0.0019 \\
CA15 & 2.83 & 12.5 & 6.62 & 0.0029 & 38.0 & 6.10 & 0.0025 \\
CA16 & 3.03 & 12.5 & 6.59 & 0.0036 & 32.5 & 5.90 & 0.0032 \\
CA17 & 3.50 & 12.5 & 6.55 & 0.0025 & 32.0 & 5.90 & 0.0022 \\
\hline CAS05 & 2.91 & 10.0 & 3.60 & 0.0028 & 45.0 & 2.90 & 0.0013 \\
CAS04 & 1.57 & 45.0 & 3.68 & 0.0015 & 60.0 & 2.70 & 0.0016 \\
CAS09 & 2.07 & 30.0 & 8.59 & 0.0016 & 30.0 & 7.85 & 0.0009 \\
CAS10 & 3.10 & 12.5 & 8.65 & 0.0023 & 30.0 & 8.00 & 0.0018 \\
\hline
\end{tabular}

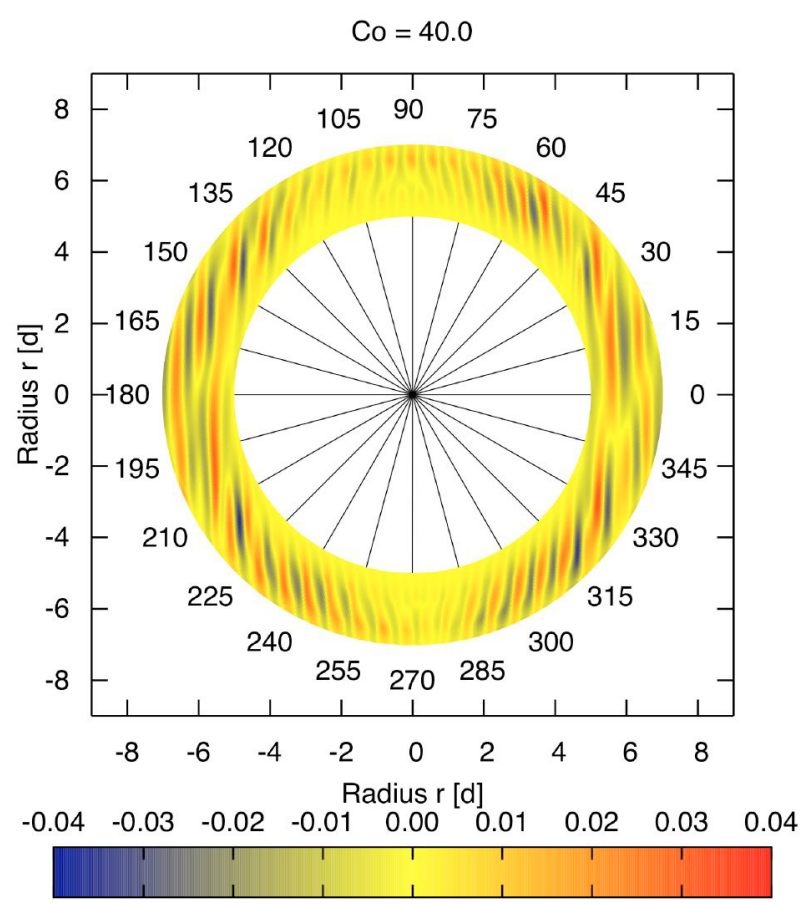

Fig. 10. Contour plot of the mean meridional velocity $\left\langle u_{\theta}\right\rangle$ from the cylinder model CA26. The flow is aligned in layers of Taylor columns.

mean meridional velocity $u_{x}$, which is very weak in the convective layer. If there is any circulation, it is rather suppressed in the rapid-rotation regime - a result which fits our findings from the cylinder simulations.

\section{Conclusion and summary}

We have performed numerical simulations of convection zones of solar-type stars with two different geometries in order to explore the influence of rotation and the resulting Reynolds stresses. We found that the horizontal transport coefficient $Q_{\theta \varphi}$ is positive on the northern hemisphere at almost all depths and latitudes, and a range of Coriolis numbers that is relevant to the solar convection zone. This agrees with results determined at the solar surface by various observers, e.g., Ward (1965), Gilman \& Howard (1984), Vršnak et al. (2003). However, in the regime of very rapid rotation the sign of $Q_{\theta \varphi}$ is reversed, and the horizontal transport is polewards. Comparing both geometries we confirmed the existence of a strong peak of $Q_{\theta \varphi}(r)$ at a low latitude and near the surface of the convection layer, but this peak

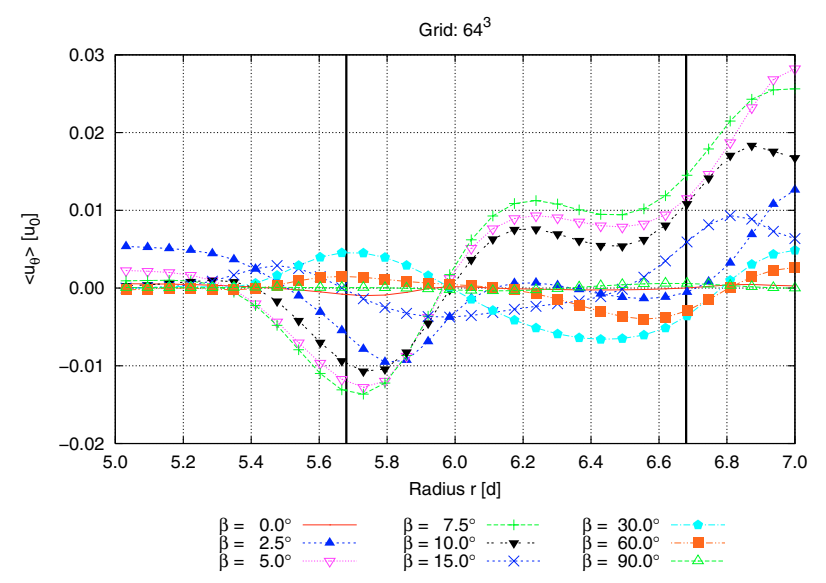

Fig. 11. Average over horizontal coordinates and time of the meridional velocity $u_{x}$ at different northern latitudes from the box model BD.

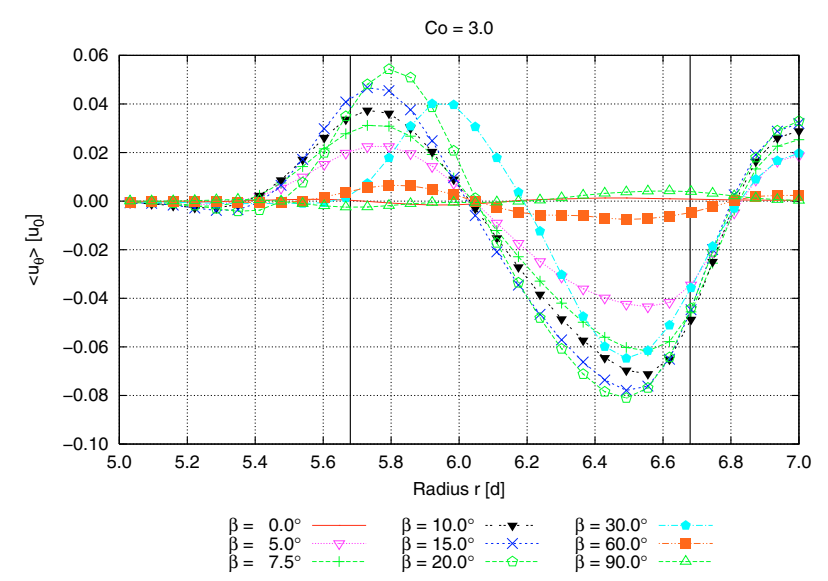

Fig. 12. Time average of the meridional velocity $u_{\theta}$ from model CA16 for different northern latitudes.

disappears in the cylinder models when the Coriolis number exceeds a value of about 4 .

Regarding the meridional flow we found that there is a circulation in the weak and moderate rotation regime, $\mathrm{Co}=0 \ldots 4$; for more rapid rotation one obtains Taylor columns in the meridional plane. The circulation is partitioned into multiple latitudinal cells for weak rotation and forms one meridional cell for moderate rotation, with counter-clockwise orientation in the convective layer.

Although the two-dimensional cylinder model has (geometric) problems in the polar regions it proves to be an efficient way 
to get information on connected flows with a high latitudinal resolution.

Acknowledgements. We gratefully acknowledge discussions with M. Ossendrijver and W. Dobler, and suggestions for improving the text by Reiner Hammer and an unknown referee. $\mathrm{CH}$ was supported by the Deutsche Forschungsgemeinschaft, and PJK acknowledges support from the Finnish graduate school for astronomy and space physics.

\section{Appendix A: The hydrodynamical stress tensor in cylindrical coordinates}

The stress tensor is symmetric; the six independent components $S_{r r}, S_{r \beta}, S_{r \phi}, S_{\beta \beta}, S_{\beta \phi}, S_{\phi \phi}$ are given by:

$$
\begin{aligned}
S_{r r} & =\frac{\partial u_{r}}{\partial r}-\frac{1}{3} \nabla \cdot \boldsymbol{u}, \\
S_{r \beta} & =\frac{1}{2}\left[\frac{1}{r} \frac{\partial u_{r}}{\partial \beta}+r \frac{\partial}{\partial r}\left(\frac{u_{\beta}}{r}\right)\right], \\
S_{r \phi} & =\frac{1}{2}\left(\frac{\partial u_{r}}{\partial \phi}+\frac{\partial u_{\phi}}{\partial r}\right), \\
S_{\beta \beta} & =\frac{1}{r} \frac{\partial u_{\beta}}{\partial \beta}+\frac{u_{r}}{r}-\frac{1}{3} \nabla \cdot \boldsymbol{u}, \\
S_{\beta \phi} & =\frac{1}{2}\left(\frac{1}{r} \frac{\partial u_{\phi}}{\partial \beta}+\frac{\partial u_{\beta}}{\partial \phi}\right), \\
S_{\phi \phi} & =\frac{\partial u_{\phi}}{\partial \phi}-\frac{1}{3} \nabla \cdot \boldsymbol{u} .
\end{aligned}
$$

\section{References}

Boussinesq, M. J. 1897, Théorie de l'écoulement tourbillonnant et tumultueux des liquides dans les lits rectilignes à grande section (Paris: Gauthier-Villars) Brandenburg, A. 2003, in Advances in nonlinear dynamos, ed. A. Ferriz Mas, \& M. Nuñez, 269

Brandenburg, A., Jennings, R. L., Nordlund, Å., et al. 1996, J. Fluid Mech., 306, 325

Caunt, S. E., \& Korpi, M. J. 2001, A\&A, 369, 706

Chan, K. L. 2001, ApJ, 548, 1102

Chandrasekhar, S. 1961, Hydrodynamic and hydromagnetic stability (Oxford: Clarendon Press)

Elliott, J. R., Miesch, M. S., \& Toomre, J. 2000, ApJ, 533, 546

Gilman, P. A., \& Howard, R. 1984, Sol. Phys., 93, 171

Gough, D., Moore, D. R., Spiegel, E. A., \& Weiss, N. O. 1976, ApJ, 206, 536

Hathaway, D. H., \& Somerville, R. C. J. 1983, J. Fluid Mech., 126, 75

Hupfer, C., Käpylä, P., \& Stix, M. 2005, Astron. Nachr., 326, 223

Käpylä, P. J., Korpi, M. J., \& Tuominen, I. 2004, A\&A, 422, 793

Kichatinov, L. L., \& Rüdiger, G. 1993, A\&A, 276, 96

Miesch, M. S., Elliott, J. R., Toomre, J., et al. 2000, ApJ, 532, 593

Ossendrijver, M. 2003, A\&ARv, 11, 287

Ossendrijver, M., Brandenburg, A., \& Stix, M. 2001, A\&A, 376, 713

Pinsonneault, M., Kawaler, S. D., Sofia, S., \& Demarque, P. 1989, ApJ, 338, 424 Pulkkinen, P., Tuominen, I., Brandenburg, A., Nordlund, Å., \& Stein, R. F. 1993, A\&A , 267, 265

Robinson, F. J., \& Chan, K. L. 2001, MNRAS, 321, 723

Rüdiger, G. 1989, Differential Rotation and Stellar Convection: Sun and solartype stars (Berlin: Akademie-Verlag)

Rüdiger, G., Egorov, P., \& Ziegler, U. 2005, Astron. Nachr., 326, 315

Schröter, E., \& Wöhl, H. 1976, Sol. Phys., 49, 19

Vršnak, B., Braǰsa, R., Wöhl, H., et al. 2003, A\&A, 404, 1117

Ward, F. 1965, ApJ, 141, 534

Weber, M., Strassmeier, K. G., \& Washüttl, A. 2005, AN, 326, 287

Zhao, J., \& Kosovichev, A. G. 2004, ApJ, 603, 776 